\title{
A Challenging Case of Postcholecystectomy Pseudoaneurysm at an Uncommon Location
}

\author{
Shayeri Roy Choudhary ${ }^{1}$ Harish Bhujade ${ }^{1}$ Pankaj Gupta ${ }^{1}$ Harjeet Singh ${ }^{2}$ Naveen Kalra ${ }^{1}$ \\ Mandeep Kang ${ }^{1}$ \\ ${ }^{1}$ Department of Radiodiagnosis and Imaging, Postgraduate Institute \\ of Medical Education and Research, Chandigarh, India \\ ${ }^{2}$ Surgical Gastroenterology, Postgraduate Institute of Medical \\ Education and Research, Chandigarh, India \\ Address for correspondence Pankaj Gupta, MD, Department of \\ Radiodiagnosis and Imaging, Postgraduate Institute of Medical \\ Education and Research, Chandigarh 160012, India \\ (e-mail: Pankajgupta959@gmail.com).
}

J Clin Interv Radiol ISVIR 2023;7:54-56.

\begin{abstract}
Hepatic artery pseudoaneurysm in postcholecystectomy choleduodenal fistula is an extremely rare but clinically significant complication. A 52-year-old male presented with hematemesis and melena several weeks after open cholecystectomy for complicated gallstone disease. Upper gastrointestinal endoscopy revealed bleeding from the choledochoduodenal fistula site. On computed tomography angiography, a small right

Keywords

- biliovascular injury

- hepatic artery pseudoaneurysm

- endovascular embolization hepatic artery pseudoaneurysm close to fistula site was identified. Endovascular embolization failed due to nonopacification of the proximal right hepatic artery. Endoscopic ultrasound-guided embolization was also unsuccessful due to the small size of the aneurysm and inadequate visualization. Following this, percutaneous $n$-butyl cyanoacrylate glue was successfully injected into the pseudoaneurysm. The patient recovered and was planned for hepaticojejunostomy to restore biliary continuity.
\end{abstract}

\section{Introduction}

Arterial injury is a relatively rare but clinically significant complication of cholecystectomy. ${ }^{1}$ Hepatic artery pseudoaneurysm requires urgent treatment, as it may lead to exsanguinating gastrointestinal bleeding. Endovascular embolization is the first choice considering the high technical and clinical success. ${ }^{2}$ However, variations in the arterial origin and course may lead to failure of embolization. Endoscopic ultrasound (EUS) is being increasingly utilized for management of pseudoaneurysms in close relation to the gastrointestinal tract lumen. ${ }^{3}$ Percutaneous embolization is the second-choice procedure that allows successful embolization in cases with failure of endovascular and endoscopic methods. ${ }^{4}$ Surgery is often reserved for unstable patients or those in whom the minimally invasive interventions fail. We present a case of postcholecystectomy right hepatic artery pseudoaneurysm at an uncommon location, near choledochoduodenal fistula, that was managed by percutaneous embolization after failure of endovascular and endoscopic procedures.

\section{Case Report}

A 51-year-old male presented with recurrent episodes of hematemesis and melena requiring multiple blood transfusions following open cholecystectomy for complicated gallstone disease at an outside hospital (2 months back). There was tachycardia and anemia (hemoglobin, $8.5 \mathrm{mg} / \mathrm{dL}$ ). Liver function tests revealed mild hyperbilirubinemia article published online February 14, 2022
DOI https://doi.org/ $10.1055 / \mathrm{s}-0042-1742728$ ISSN 2457-0214.
(C) 2022. Indian Society of Vascular and Interventional Radiology. All rights reserved.

This is an open access article published by Thieme under the terms of the Creative Commons Attribution-NonDerivative-NonCommercial-License, permitting copying and reproduction so long as the original work is given appropriate credit. Contents may not be used for commercial purposes, or adapted, remixed, transformed or built upon. (https://creativecommons.org/ licenses/by-nc-nd/4.0/)

Thieme Medical and Scientific Publishers Pvt. Ltd., A-12, 2nd Floor, Sector 2, Noida-201301 UP, India 


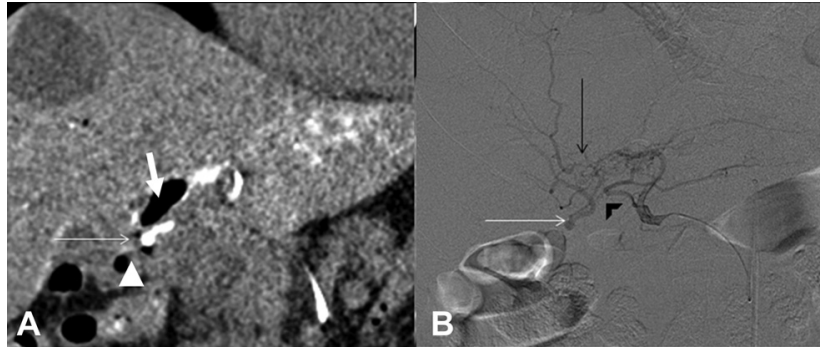

Fig. 1 Coronal computed tomography angiography (A) image showing right hepatic artery pseudoaneurysm (arrow) in close relation to choledochoduodenal fistula site (arrowhead). Note the gas within the bile duct (short arrow). Celiac arteriogram (B) showing right hepatic artery pseudoaneurysm (arrow) that is filling from the small arterial collaterals from left hepatic artery (black arrow). Right hepatic artery is not visualized proximally (arrowhead).

(total/conjugated bilirubin, 1.8/0.8 mg/dL), and elevation of alkaline phosphatase ( $520 \mathrm{U} / \mathrm{L})$. Upper gastrointestinal tract endoscopy showed a small opening at D1-D2 junction from which fresh blood was oozing. Computed tomography (CT) angiography revealed a small contrast filled outpouching $(2.5 \times 2.5 \mathrm{~mm})$ arising from the right hepatic artery adjacent to the choledochoduodenal fistula site (-Fig. 1A). Biliary dilatation and multiple cholangitic abscesses in the right lobe of liver were also seen. A diagnosis of postcholecystectomy biliovascular injury with right hepatic artery pseudoaneurysm was considered. Celiac axis angiogram showed opacification of the left hepatic artery (-Fig. 1B). The proximal right hepatic artery was not visualized suggesting iatrogenic thrombosis. The right hepatic artery pseudoaneurysm filled retrogradely from the left hepatic artery branches. The microcatheter could not be negotiated from the small left hepatic artery branches into the pseudoaneurysm and hence the endovascular procedure was abandoned. EUS-guided transmural embolization was planned and patients was shifted to endoscopy suite. On EUS, the pseudoaneurysm was not well visualized due to its small size and location near the choledochoduodenal fistula site. Percutaneous embolization was performed as a salvage nonsurgical procedure. Percutaneous transhepatic sac embolization was done using a 22-gauge Chiba needle $(15 \mathrm{~cm}$ ) and $1 \mathrm{~mL}$ mixture of $n$-butyl cyanoacrylate (NBCA) glue and ethiodized oil (Lipiodol Ultra Fluid, Guerbert, Villepinte, France) in a 1:1 ratio under fluoroscopic guidance (-Fig. 2A). There was opacification

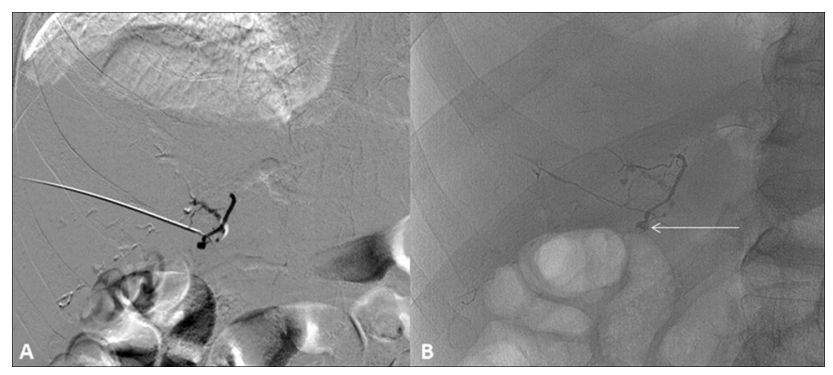

Fig. 2 Fluoroscopy-guided cyanoacrylate glue injection into the pseudoaneurysm (A). Post procedure single-shot fluoroscopic image showing glue cast in the pseudoaneurysm sac (arrow, B) as well as in the feeding artery.

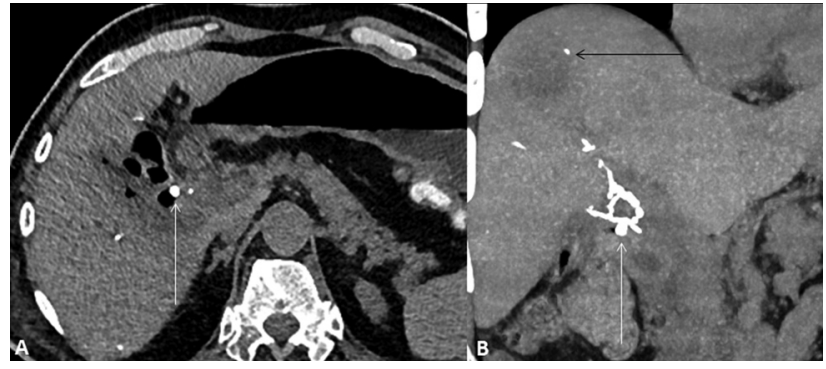

Fig. 3 Post procedure non-contrast computed tomography abdomen axial (A) and coronal maximum intensity projection image (B) after 24 hours showing hyperdense cyanoacrylate glue cast within the sac and surrounding arteries (arrows, $\mathbf{A}$ and $\mathbf{B}$ ). Nontarget embolization is seen in the right lobe of liver due to aggressive embolization (black arrow, B). However, the patients tolerated the procedure well.

of the sac as well as the artery (-Fig. 2B). A noncontrast CT abdomen confirmed the presence of hyperdense cast within the pseudoaneurysm ( $\mathbf{- F i g . 3 A}$ and $\mathbf{B}$ ). There was resolution of hematemesis and melena within 12 hours. The patient tolerated the procedure well and had no fresh complaints. He was discharged in a stable situation and was planned for an elective hepaticojejunostomy.

\section{Discussion}

The most common type of bilioenteric fistula is cholecystoduodenal fistula that occurs in patients with chronic cholecystitis. ${ }^{5}$ Choledochoduodenal fistula occurs in patients with peptic ulcer disease or impacted common bile duct stones. Spontaneous choledochoduodenal fistula due to iatrogenic bile duct injury during laparoscopic cholecystectomy is rare. ${ }^{6}$ Gastrointestinal bleeding may be one of the manifestations of choledochoduodenal fistula. ${ }^{5}$ However, to the best of our knowledge, pseudoaneurysm adjacent to choledochoduodenal fistula following laparoscopic cholecystectomy has not been previously reported.

Postcholecystectomy hepatic artery pseudoaneurysms are rare, but clinically significant. They may occur as an isolated complication, but more commonly occur in association with iatrogenic bile duct injuries. Overall, the incidence of vascular complications in bile duct injuries is up to 25 to $35 \%{ }^{7}$ Hepatic artery pseudoaneurym (most commonly from the right hepatic artery, common hepatic artery or cystic artery) is seen in about $0.6 \%$ of postcholecystectomy patients. ${ }^{7}$ The presenting symptoms are right upper quadrant pain, gastrointestinal bleeding, and obstructive jaundice. ${ }^{7}$ A high degree of clinical suspicion of biliovascular injury is warranted in patients with these symptoms who have undergone recent cholecystectomy. CT angiography is an ideal investigation in the emergent management of suspected biliovascular injury.

The treatment of choice for hepatic artery pseudoaneurysm is endovascular embolization. ${ }^{2}$ EUS-guided thrombin injection is a relatively novel approach in mesenteric pseudoaneurysms that are not accessible endovascularly. ${ }^{3}$ In our case, an attempt was made to approach the sac under EUS guidance; however, due to the subcentimetric size of the sac 
and complex anatomy of the region, the procedure was unsuccessful. Percutaneous embolization of hepatic artery pseudoaneurysms is a salvage procedure following an unsuccessful endovascular and EUS-guided embolization. The procedure must be carried out with utmost care to avoid leakage of the embolizing agent into the arterial circulation. The addition of lipiodol acts as a carrier, which aids in smooth and controlled injection of the embolic agent. Lipiodol also confers radio-opacity to the embolization agent and helps to visualize the embolization process under fluoroscopy. Glue polymerizes upon contact with ions in the blood, forming a cast within the vessel lumen. ${ }^{8}$ The complications of percutaneous NBCA injection include nontarget embolization (which may lead to massive hepatic necrosis) or pseudoaneurysm sac rupture. Inadvertent injection into the bile ducts can cause cholangitis. Injection into the portal or hepatic veins may cause venous thrombosis. Fortunately, these complications are extremely rare in expert hands. Treatment with percutaneous thrombin is safer, as it acts by augmenting the native coagulation system by forming a fibrin clot within the pseudoaneurysm lumen. The clot causes stasis and acts as a mesh for endogenous platelet aggregation and thrombus formation. However, use of thrombin is associated with increased risk of recanalization. ${ }^{9}$

In conclusion, pseudoaneurysm at the choledochoduodenal fistula site following cholecystectomy is an extremely rare but catastrophic complication that can be successfully managed with minimally invasive techniques.

Conflict of Interest

None declared.

\section{References}

1 Gorsi U, Gupta P, Kalra N, et al. Multidetector computed tomography evaluation of post cholecystectomy complications: a tertiary care center experience. Trop Gastroenterol 2015;36(04): 236-243

2 Sagar S, Soundarajan R, Gupta P, et al. Efficacy of endovascular embolization of arterial pseudoaneurysms in pancreatitis: a systematic review and meta-analysis. Pancreatology 2021;21 (01):46-58

3 Rai P, Kc H, Goel A, Aggarwal R, Sharma M. Endoscopic ultrasoundguided coil and glue for treatment of splenic artery pseudoaneurysm: new kid on the block!. Endosc Int Open 2018;6(07): E821-E825

4 Vyas S, Ahuja CK, Yadav TD, Khandelwal N. Emergency ultrasound-guided percutaneous embolization of post-traumatic bleeding hepatic artery pseudoaneurysms. Minim Invasive Ther Allied Technol 2012;21(05):372-376

5 Crespi M, Montecamozzo G, Foschi D. Diagnosis and treatment of biliary fistulas in the laparoscopic era. Gastroenterol Res Pract 2016;2016:6293538

6 Yilmaz S, Akici M, Okur N, Türel S, Erşen O, Şahin E. Spontaneous postoperative choledochoduodenal fistula due to bile duct injury following laparoscopic cholecystectomy. Int J Surg Case Rep 2016; 25:199-202

7 Stewart L, Robinson TN, Lee CM, Liu K, Whang K, Way LW. Right hepatic artery injury associated with laparoscopic bile duct injury: incidence, mechanism, and consequences. J Gastrointest Surg 2004;8(05):523-530, discussion 530-531

8 Yadav RR, Boruah DK, Bhattacharyya V, et al. Percutaneous direct needle puncture and transcatheter $\mathrm{N}$-butyl cyanoacrylate injection techniques for the embolization of pseudoaneurysms and aneurysms of arteries supplying the hepato-pancreato-biliary system and gastrointestinal tract. J Clin Imaging Sci 2016;6:48

9 Kang SS, Labropoulos N, Mansour MA, et al. Expanded indications for ultrasound-guided thrombin injection of pseudoaneurysms. J Vasc Surg 2000;31(02):289-298 